\title{
Mutterlandpop. Lokale Markierung Und ENTGRENZUNG MUSIKALISCHER DARBIETUNGEN AUF UKRAINISCHEN FEIERTAGEN
}

\section{Christian Diemer}

Die Frage der (Selbst-)Verortung der Ukraine - politisch, aber auch kulturell - stellt sich nicht erst seit Ausbruch des bewaffneten Konflikts ${ }^{1}$ in der Ostukraine mit Vehemenz. Dieser Aufsatz untersucht die Verwendung und Funktion von Musik an ukrainischen Feiertagen vor dem Hintergrund der Frage, wie und durch welche musikalischen Repräsentationen regionale Markierung einerseits erzeugt bzw. andererseits verwischt und entgrenzt wird. Dazu habe ich die Programmgestaltung mehrerer Feiertage in unterschiedlichen Gegenden der Ukraine ethnographisch dokumentiert und ausgewertet. Das Verhältnis von Lokalität und Traditionalität ist hierbei nicht von vornherein gleichzusetzen, wobei gleichwohl beide Aspekte in einem denkbar engen Verhältnis zueinander stehen: Traditionelle Musik und Kultur erscheinen in der Ukraine als das Medium, um lokalen Bezug auszudrücken und Identitätskonstruktion zu bewerkstelligen (vgl. hierzu anschaulich Andruchovyč 2003: 26ff.; Andruchovyč 2004: 25ff.).

\section{Zum Forschungs-Arrangement}

Die Untersuchung basiert auf ethnographischer Feldforschung, die ich seit 2009/10 und schwerpunktmäßig ab 2013 in der Ukraine durchgeführt habe. Die Feldforschung fand statt im Sinne teilnehmender Beobachtung, ohne

1 Völkerrechtlich ist der Terminus des »bewaffneten Konflikts « oder des »internationalen bewaffneten Konflikts « zutreffend (Wissenschaftliche Dienste des Deutschen Bundestags 2010) - letzterer dann, wenn man der Einschätzung etwa der Bundesregierung folgt, dass das Kriegsgeschehen in der Ostukraine maßgeblich von russischen Freischärlern, regulären Soldaten sowie militärischer Aufrüstung über die unkontrollierte ukrainisch-russische Grenze beeinflusst wird. Russland dementiert dies. 
dass eine Beeinflussung der Akteure durch den Beobachter entstand. Die Beobachtungen wurden ergänzt durch qualitative Interviews, Hintergrundgespräche und kontextuelle Einordnungen mit Betroffenen.

Forschung fand in unterschiedlichen Regionen der Ukraine statt. Dies erschien wichtig, um das verbreitete und durch die konflikthaften Ereignisse scheinbar bestärkte Paradigma einer inner-ukrainischen Spaltung nicht ungeprüft ins Forschungsdesign zu übernehmen (vgl. stellvertretend die Theorie der »zwei Ukrainen« bei Rjabčuk 2005 und kritisch dazu Jilge 2005). Schwerpunktmäßig habe ich Forschung durchgeführt in folgenden oblasti (Regionen) $)^{2}$ :

- Černivci / Czernowitz (Südwestukraine, 40 Kilometer von der rumänischen Grenze entfernt),

- L'viv / Lemberg (Westukraine, 70 Kilometer von der polnischen Grenze entfernt),

- Žytomyr (Zentralukraine, 140 Kilometer westlich von Kyïv),

- Charkiv / Har'kov (Ostukraine, 40 Kilometer von der russischen Grenze entfernt).

L'viv (734.000 Einwohner) gilt als Zentrum des ukrainischen Nationalstolzes. Dementsprechend habe ich dort den ukrainischen National- und Unabhängigkeitsfeiertag dokumentiert.

Im ostukrainischen oblast' Charkiv galten meine Forschungen den Stadtund Befreiungsfesten der Provinzstadt Lozova (56.910 Einwohner) und des nahegelegenen Dorfs Sachnovščyna (7.937 Einwohner). Die dortigen Feierlichkeiten, die zugleich Stadtfest (den' goroda) sind, stehen im Zeichen des Gedenkens der Befreiung von der deutschen Wehrmacht und der Verdienste der Veteranen.

Im westukrainischen Dorf Vaškivci (5.660 Einwohner, oblast' Černivci) empfahl sich das in der ukrainisch-rumänischen Grenzregion verbreitete Malanka-Fest: Die Neujahrs-Feier nach julianischem Kalender verbindet sich dort mit Weihnachts-, Dreikönigs- und Karnevals-Elementen und genießt besonders in ihrer Ausformung in Vaškivci den Ruf besonderer Traditionsverbundenheit. Nach den jeweiligen Feiern auf den Dörfern treffen die Akteure beim großen Malanka-Festival in der Regionshauptstadt Černivci zusammen.

In der ehemaligen Industriestadt Korosten' (66.000 Einwohner), gelegen im zentralukrainischen oblast' Žytomyr, ist das traditionelle Kartoffelpuffer-

2 Das ukrainische Staatsgebiet ist administrativ in 24 oblasti aufgeteilt; hinzu kommen die Autonome Republik Krym sowie zwei "Städte mit besonderem Status« (Kyïv und Sevastopol). Seit März 2014 hat die Ukraine keine Kontrolle mehr über die Autonome Republik Krym und die Stadt Sevastopol. Russland sieht diese seit 21.3.2014 als der Russländischen Föderation zugehörig an. 
fest (prazdnik deruniv, zu Ehren des traditionellen ukrainischen Gerichts) ein alljährlicher Höhepunkt von großer Bedeutung für das Stadtmarketing der von Strukturproblemen betroffenen Stadt. Damit sind in geographischer Hinsicht Schwerpunkte in der West-, Zentral- und Ostukraine abgedeckt.

Aufgrund des Forschungsarrangements lassen sich auch in zeitlicher Dimension bedingt Aussagen treffen, insbesondere über etwaige Veränderungen in Folge der Majdan-Revolution und des Ausbruchs des bewaffneten Konflikts in der Ostukraine. So waren zum Zeitpunkt der Dokumentationen des Nationalfeiertags in L'viv am 24.8.2013 und des Stadtfestes in Sachnovščyna am 17.9.2013 noch keinerlei Anzeichen eines baldigen Umsturzes zu erkennen, von gelegentlich geäußerter Empörung über den unter meinen ukrainischen Gesprächspartnern als Kleptokraten verschrienen Vyktor Janukovyč abgesehen. Im darauffolgenden Jahr hingegen feierte das postrevolutionäre L'viv die Unabhängigkeit von Russland, welche im selben Moment in den östlichen Regionen des Landes ernstlich gefährdet war und nach wie vor ist. Das ostukrainische Lozova fand sich 2014 beim 71-jährigen Gedenken an seine Befreiung vom Zweiten Weltkrieg auf einmal nur noch 90 Kilometer vom Schauplatz neuerlicher Kriegshandlungen entfernt.

Auch die Malanka-Feste in Vaškivci und Černivci vom 13.-15.1.2014 fielen zeitlich gerade noch vor das entscheidende Wiederaufflammen der Majdan-Proteste in der Hauptstadt Kyïv und auch in der nahegelegenen Regionshauptstadt Černivci (242.300 Einwohner). Die Proteste waren am 20.11.2013 durch die Nicht-Unterzeichnung des EU-Assoziierungsabkommens durch Vyktor Janukovyč ausgelöst worden. Über die orthodoxen Weihnachts- und Neujahrsfeierlichkeiten schienen sie weitgehend zum Erliegen gekommen zu sein. Erst danach nahmen sie wieder Fahrt auf, um am 22.1.2014 mit dem ersten Toten auf dem Kiewer Majdan eine neue Stufe der Eskalation zu erreichen. Am 13./14.1.2015 hingegen, zum Zeitpunkt des Malanka-Festes ein Jahr später, hatte der Krieg im Osten selbst diese äußerste westliche Region der Ukraine erreicht, in Gestalt einer Bombendrohung in Černivci.

Die Beobachtungen und Interpretationen erheben nicht den Anspruch, geographische Unterschiede und zeitlich verlaufende gesellschaftliche Transformationsprozesse in der Ukraine erschöpfend oder repräsentativ abzubilden. Dies ist allein aufgrund logistischer Einschränkungen nicht möglich, da eine persönliche Dokumentation zweier gleichzeitiger Feiertage an verschiedenen Orten nicht möglich war.

Bei den parametrischen Einbettungen und Korrelationen handelt es sich um Interpretationsversuche, die durch Hintergrundgespräche mit Einheimischen abgesichert oder - zu deren womöglich differenten Einschätzungen in Relation gesetzt wurden. Es versteht sich von selbst, dass keiner der 
Parameter monodimensional bzw. monokausal vorliegt, es müssen komplexe Überlagerungs- und Auslöschungseffekte angenommen werden.

Dementsprechend nimmt die detaillierte Beschreibung der jeweiligen Beobachtungen im Rahmen des Aufsatzes großen Raum ein. Erst im zweiten Schritt werden Thesen zur Subsumierung und Deutung der Befunde vorgestellt. Aussagekräftig ist die Beschreibung nicht zuletzt aufgrund der Vielfalt der unterschiedlichen Messpunkte - in geographischer und zeitlicher Hinsicht wie mit Blick auf das komplexe Geflecht weiterer Faktoren. Dies erscheint als notwendige Voraussetzung dafür, Aussagen jenseits der tautologischen Vorfestlegungen treffen zu können, die nicht nur den politischen, sondern teilweise - selbst in der Ukraine - auch den wissenschaftlichen Diskurs beherrschen. Nicht zuletzt um diese zu korrigieren oder zumindest zu relativieren und erheblich zu differenzieren, erscheinen die vorliegenden Beobachtungen hilfreich und wichtig.

\section{Forschungszyklus 2013/14 (vor Majdan-Umsturz und Ausbruch des bewaffneten Konflikts)}

a) L'viv, Nationalfeiertag $(24.8 .2013)$

Das musikalische Hauptprogramm des Nationalfeiertags in L'viv 2013 wurde auf einer großen Bühne auf dem zentralen Marktplatz der Stadt dargeboten. Es bestand vor allem in einer langen Abfolge von Auftritten von so genannten Folklore-Kollektiven (folklor'nij kollektiv oder folklor'nij ansambl' in ihrer ukrainischen Selbstbezeichnung), insbesondere Kinder-Tanzgruppen, die in traditionellen Kostümen zu zumeist von Band abgespielter Folklore-Musik ${ }^{3}$ traditionelle Tänze ${ }^{4}$ aufführten. Auf die Herkunft der jungen »Talente« aus

3 In der Ukraine ist der Begriff »folklor'« nicht abwertend konnotiert, sondern als Sammelbezeichnung für mehr oder weniger authentische Darbietungen traditioneller Musik in Gebrauch. Außerdem ist von Volks- oder volkstümlicher Musik die Rede (»narodna muzyka«). Die objektivere Bezeichnung als »traditionelle Musik« (»tradicijna muzyka«) hingegen löst im alltagssprachlichen Kontext die Nachfrage aus, was genau gemeint sei.

4 Die Einordnung sowohl von Choreographie als auch Musik in eine solche Kategorie ist in diesen Fällen besonders problematisch, da es sich hier eben nicht um ethnologisch informierte Darbietungen mit irgendeiner Form von Authentizitätsanspruch handelt. Vielmehr sind die Beiträge sicherlich als traditionell inspiriert anzusehen und werden auch als solche bezeichnet und verstanden, entfernen sich aber in vieler anderer Hinsicht weit von tatsächlicher traditioneller Musikpraxis. Dies beginnt mit der Kombination mit traditionsfremden Instrumenten und reicht über das Faktum einer einstudierten Choreographie bis hin 
»unserer Region« wurde von der Moderation stets verwiesen. Dazwischen hatte das Erwachsenen-Folklore-Ensemble Rusalka aus Kanada mit einer professionellen Choreographie in traditionellen ukrainischen Kostümen einen umjubelten Gastauftritt.

Zugleich fanden sich Präsentationen ausdrücklicher Popmusik ins Programm eingestreut, z.B. der (gecoverte) Song »Zliva ta Polum'ja« der Kiewer Popsängerin Nina Matvienko (*1981), oder der viermalige Auftritt des Lemberger Popsängers Ihor Netljuch (*1983), teilweise begleitet von pantomimisch agierenden Tanzpartnerinnen.

Gegen Abend des Nationalfeiertags fächerte sich die stilistische Bandbreite noch weiter auf: Die Lemberger Philharmonie gab Werke von Vivaldi, Mozart und Johann Strauß (Sohn). Nach dem offiziellen Ende der Darbietungen auf der Hauptbühne erklang hinter dem Rathaus zu Playback Jazz-Saxophon (u.a. »Lily Was Here « von Dave A. Stewart und Candy Dulfer), während auf der anderen Seite des Rathauses zu Salsa-Musik getanzt wurde (u.a. »El Negre Vielve a la Habana« von Issac Delgado). Letzteres ist eine Initiative, die unabhängig vom Nationalfeiertag in der Sommerzeit allabendlich fortgeführt wurde.

Dies wäre alles für sich vielleicht noch nicht sonderlich bemerkenswert; ein Nationalfeiertag hat die Geschmäcke vieler Zuhörergruppen zu bedienen, warum auch nicht von ein und derselben Hauptbühne aus und in bunter Durchmischung der Abfolge. Von gesteigertem Interesse sind daher einige Darbietungen, innerhalb derer Pop- und Folklore-Elemente sich überlagern, und zwar auf jeweils recht unterschiedliche Weise.

So wurden etwa einige Popsongs gewissermaßen additiv, ohne Eingriff in ihre inhaltliche oder musikalische Faktur und auch mit nur geringer Veränderung ihrer performativen Rahmenbedingungen, mit zusätzlichen traditionellen Kodierungen versehen. Der bekannte Popsong »Za lizamy, horamy« (»Hinter Wäldern und Bergen«) von Zlata Ognevič (*1986) wurde vor Playback durch eine junge Solistin in traditionellem ukrainischem Kostüm dargeboten. Der Popklassiker »Kraj, mij ridnij kraj« (»Land, mein Mutterland«, 1983) von Sofija Rotaru (*1947) wurde von einem Mädchen in einer Kombination aus traditioneller ukrainischer Schürze und farblich abgestimmten Lackstiefeln vorgetragen. Ungeachtet der traditionellen Anleihen bei der Kostümierung waren Bühnenaktion und Habitus beider Sängerinnen - im Rahmen ihrer Möglichkeiten und Bühnenerfahrung - vollständig an Popvorbildern orientiert, etwa was einschlägige Armgesten oder das bewusst inszenierte Herumlaufen auf der Bühne betrifft.

zu grundsätzlichen performativen Rahmenbedingungen: Musik vom Band, Tanzende auf einer Bühne usw. 
Im Gegensatz dazu wurde der Pophit »Divčina Vesna« (»Frühlingsmädchen«, 2004) von Natalija Bučins'ka (*1977) ohne Live-Solosängerin komplett von Band eingespielt und durch eines der Kinder-Tanzkollektive aufgeführt. Traditionelle Montur und Choreographie unterschieden sich dabei nicht von den vorangehenden Tanzaufführungen zu instrumentaler Folkloremusik.

Einschränkend muss gesagt werden: Schon bei den zugrundeliegenden Original-Popsongs kann man davon ausgehen, dass sie eine Affinität zur Inszenierung lokaler und traditioneller Elemente bereithalten, etwa in ihren Themen und Texten (Natur, Frühling, Mutterland). Nicht zuletzt aufgrund dieses Umstands sind sie im Musikablauf des Nationalfeiertags als essentieller Bestandteil programmiert.

Auch mag die traditionelle Kostümierung der Sängerinnen grundsätzlich nicht überzubewerten sein. In den letzten Jahren ist es in der Ukraine modisch geworden, ansonsten zeitgenössische Kleider z. B. mit den traditionellen bestickten Hemden (vyšyvanki) zu kombinieren. Am Nationalfeiertag tragen besonders viele Ukrainerinnen und Ukrainer diese Hemden. In L'viv waren am Nationalfeiertag 2013 sogar die Steinskulpturen der die Ecken des Marktplatzes zierenden Brunnen in überdimensionale vyšyvanki eingekleidet. - Gleichwohl zeugen die beschriebenen Befunde allgemein von einer hohen Durchlässigkeit bezüglich lokal bzw. traditionell markierter sowie globalisierter bzw. zeitgenössischer Elemente.

Einen besonderen Fall im Programm stellte der Beitrag der jungen Lemberger Popsängerin Jana Hurul'ova (*1997) dar (vgl. Hurul'ova 2013a). Es handelte sich um eine Bearbeitung des traditionellen ukrainischen Liedes »Letyla Zozulja« (»Es flog der Kuckuck«) durch den Arrangeur Danyl Charčev. Die im traditionellen Original getragene, klagende Liedmelodie ist in der Neufassung mit hauptsächlich elektronisch instrumentierten, stampfenden Powerchord-Riffs unterlegt. In einem Intro vor Einsetzen des Beats erzeugen streicherähnliche synthetische Orgelpunkte und Akkordflächen sowie Chimes eine geheimnisvolle Spannung. Getrommel und ein im Hintergrund erklingendes Zymbal mischen eine archaisch-exotische Konnotation bei. Das Zymbal mag an die traditionelle Provenienz der »Zozulja«-Melodie gemahnen. Allerdings ist dieses Instrument in traditionellen Versionen des Liedes nicht anzutreffen; die Rückbindung greift im Grunde sachlich ins Leere. Zwischen dritter und vierter Liedstrophe ist in der Bearbeitung ein modulierendes Interlude eingefügt, mit dem über ein Instrumentalsolo im Zurna-Sound eine weitere Weltmusik-Farbe ins Spiel gebracht wird - allerdings (erneut) mitnichten eine ukrainische. ${ }^{5}$

5 Das Phänomen der Zusammenstellung von Instrumenten aus unterschiedlichsten Musikkulturen verkörpert auf besonders erstaunliche Weise die - wie sie sich 
Die Sängerin intoniert schon während des Intros die traditionelle Zozulja-Melodie, mit stark behauchter Popstimme. Mit dem Einsatz von Beat und Riff passt sich die Stimme dem harten Duktus an, stampft Silben und Melodieschritte mit und kommt mit rockigem Sound daher. Interessanterweise zeigt der Stimmklang Hurul'ovas eine gewisse Unstetheit, die neben dem stilistischen Hybrid der Bearbeitung vielleicht auch der geringen Erfahrung der jungen Sängerin geschuldet ist. In einigen Silben nähert ihre Stimme sich jedenfalls dem scharfen, gepressten Klang an, der die traditionelle ukrainische Gesangsweise kennzeichnet: Eine Vorstellung der Klangerzeugung im vordersten Bereich der Mundhöhle und die durchdringende Stimmkraft der Sängerinnen begünstigen die Entstehung eines intensiven und sozusagen »zudringlichen « Obertonspektrums. Dasselbe gilt für einige aufwärtsjuchzende Glissandi Hurul'ovas beim Absingen der Phrasen - Anjottieren, Ausjuchzen und Tremolieren gelten als Charakteristika des traditionellen ukrainischen Stimmklangs. ${ }^{6}$

\section{b) Sachnovščyna, Stadtfest $(17.9 .2013)$}

Blickt man auf das Stadt- und Befreiungsfest des 1.000 Kilometer östlich von L'viv gelegenen Dorfes Sachnovščyna, so ergibt sich eine noch komplexere Gemengelage unterschiedlichster musikalischer Stilistiken und lokaler Markierungen. Der Stadtfeiertag beginnt traditionell mit einer feierlichen Blumenniederlegung am Denkmal der Gefallenen des »Großen Vaterländischen Krieges« (Zweiter Weltkrieg). Von dort geht es über einen von Ständen gesäumten Fußweg zum eigentlichen Festakt und Konzert im örtlichen Kultur-

selbst bezeichnet - »Ethnochaos«-Gruppe Dachabracha (Dachabracha 2015b). Das experimentelle Projekt hat sich der Schaffung von nichts weniger als einer neuen ukrainischen Identität und Mythologie verschrieben (Dachabracha 2015a: $/ \mathrm{ukr} / \mathrm{projects}$ ). Seine vier Musiker verwenden neben traditionellem obertonreichem Gesang und Akkordeon Instrumente wie Mundharmonika, Violoncello, Posaune, Dudelsack, Digeridoo, belarusische žalijka, arabische darbūka, indische tablā (Dachabracha 2015c). - Es bleibt im Einzelfall zu untersuchen, inwieweit dadurch multiple lokale Markierungen stattfinden oder inwieweit die jeweils an die Instrumente gekoppelten lokalen Markierungen nivelliert werden bzw. im Sinne einer hybriden ukrainischen Neulokalisierung eine Umkodierung erfahren.

6 Information von Mar'jana Sadovs'ka bei einem Workshop in der Berliner Philharmonie am 26.4.2014. Mar'jana Sadovs'ka $\left({ }^{*} 1972\right)$ ist eine ukrainische Sängerin, Musikerin, Komponistin und Schauspielerin, die sich intensiv mit der Erforschung und Neubelebung traditionellen ukrainischen Liedguts beschäftigt. Zugleich experimentiert sie mit transkulturellen und genreüberschreitenden Elementen, etwa mit ihrer in Deutschland ansässigen Jazz-Band Borderland oder dem US-amerikanischen Kronos Quartet. 
palast. Den Nachmittag verbringen die Sachnovščynaer an den Ständen und auf dem Leninplatz, auf dem, unter dem Denkmal des sowjetischen Führers, verschiedene Aktivitäten und Wettbewerbe für Kinder stattfinden. Der Abend bringt mit einem Open-Air-Konzert an gleicher Stelle einen weiteren Höhepunkt.

Rückgrat des Feiertags 2013 war die örtliche Militärkapelle. Die Blumenniederlegung am Denkmal begleitete sie mit sowjetischen Märschen, aufgelockert von vereinzelten getragenen Bigband-Standards. Im Konzert im Kulturpalast wechselten - ähnlich wie auf dem Lemberger Nationalfeiertag Kindertanz-Einlagen zu eingespielter Folkloremusik mit Popsongs zu Playback, dargeboten von lokalen Jugendlichen. An den farbenprächtigen Ständen zwischen Kulturpalast und Leninplatz präsentierten sich die Frauen der umliegenden Dörfchen in traditionellen Kostümen mit traditionellen, selbst zubereiteten Gerichten, Selbstgebranntem (samohon) und mehr oder minder spontanem traditionellen ukrainischen Gesang. Überwiegend handelte es sich bei den Frauen um miteinander bekannte Nachbarinnen aus dem jeweiligen Dorf, nicht um etablierte Ensembles. Sie sangen, im Halbkreis stehend, von Bekannten umringt oder verstärkt, meist instrumental unbegleitet und ohne festgelegtes Programm. In direkter Hör- und Sichtweite der Frauen spielte die Militärkapelle in Uniform angloamerikanische Klassiker wie Quincy Jones' »Soul Bossa Nova« (1962), besser bekannt als Titelmelodie des Films Austin Powers (1997), oder ein Bigband-Arrangement von Deep Purples »Smoke On The Water $«$ (1972).

Ein weiterer, nachmittäglicher Auftritt der Militärkapelle auf dem Leninplatz wurde verstärkt durch ein Drumset und eine E-Gitarre. Neben sowjetischen Märschen wurden in ebendieser Abfolge gespielt:

- das ukrainische Volkslied »Oj, ty ničen'ko (wörtlich »Oi, du bist das Nächtlein«) - mit klassisch ausgebildetem Solosänger in Uniform und Bigband-Begleitung -,

- erneut »Soul Bossa Nova« / Austin Powers,

- James Browns »| Got You (I Feel Good)« (1965) mit einer jungen PopSolosängerin mit weißen Lackstiefeln,

- zwei akrobatische Musik- und Turneinlagen der Militärkapelle,

- Sofija Rotarus »Kraj « (1983) - das auch auf dem Lemberger Nationalfeiertag erklang - in Bigband-Arrangement und mit derselben Popsängerin.

Das Kinderprogramm auf dem Leninplatz bot beliebte Talent-Wettbewerbe an. Im Rahmen von Karaoke-Tanzimprovisationen wurde u.a. Psys »Gangnam Style « (2012) aufgelegt. Für das später am Abend den Platz beschallende Konzert waren eine Popsängerin und, nach einem Feuerwerk, noch eine 
Rockband aus der Provinzhauptstadt Charkiv eingeladen. Die Popsängerin stimmte u.a. nochmals »Kraj« von Sofija Rotaru an.

\section{c) Vaškivci, Malanka-Fest (13./14.1.2014)}

Nicht nur an von städtischer Seite eher homogen programmierten Feiertagen wie dem Nationalfeiertag in L'viv oder dem Stadtfest in Sachnovščyna, auch an anderen, stärker aus individuellen Beiträgen zusammengesetzten Feiertags-Veranstaltungen in der Ukraine zeigt sich eine große Flexibilität im Umgang mit unterschiedlichen Genres - und damit auch unterschiedlichen Graden und Qualitäten lokaler Kodierung. Zum Malanka-Fest in Vaškivci am 13./14.1.2014 fuhren phantasievoll dekorierte Karnevalswagen durch den Ort, samt üppiger Boxenanlagen. Hauptsächlich schallte lautstark ukrainische und russische Popmusik von innen herab, freilich nicht selten folkloristischen Einschlags. Ein feuerspuckender Death Metal-Wagen erregte besonderes Aufsehen. Während das abendliche Singen der traditionellen ukrainischen Weihnachtslieder (Koljaden) ausschließlich den um die Häuser ziehenden Kindern vorbehalten schien, fielen unter den live agierenden Erwachsenen in erster Linie vereinzelte rumänische Musikanten auf.

Indessen ist das Malanka-Karnevalsgeschehen selbst von traditionellen wie zeitgenössischen Aspekten durchdrungen. Horden von kräftigen jungen Männern, als wilde, zottelige Bären verkleidet, verbreiten wie in der alemannischen Fastnacht rhythmischen Schellenklang - und außerdem Angst und Schrecken unter den Mädchen, auf die sie sich in konzertierten Aktionen mit Gebrüll stürzen, um sie hoch in die Luft zu werfen und wieder aufzufangen. Frauen wie auch Männer verkleiden sich mit dicken Kissen und knollennasigen Masken als altertümliche Bauernweiber. Demgegenüber stehen Kohorten von angriffslustigen Friseuren, Ärzten, transvestierenden Krankenschwestern, Scream-Masken, Zombies mit heraushängenden GummiPenissen etc. Das Spiel mit Geschlechtsumkehrungen und Homosexualität, das ansonsten in der Ukraine weitgehend tabuisiert ist, folgt westlicher Liberalität wie zugleich althergebrachter Karnevalslogik (vgl. Mezger 1984).

Außer Gebrüll und Schellenklang ist auf den unbeleuchteten Dorfstraßen, Feldwegen und Ackersäumen akustisch das Tröten verschiedenster trompetenartiger Instrumente vorherrschend. Einerseits bedeuten sie zwar eine Reminiszenz an die langgezogenen Trembiten der nahegelegenen Karpaten. Andererseits aber werden sie ganz pragmatisch durch Trompeten, Bügelhörner und Plastik-Vuvuzelas ersetzt. Die Vuvuzelas, im Zuge der Südafrika-WM 2002 als zunächst lokale Bezugnahme auf das Gastgeberland vermarktet, dann binnen kürzester Zeit nahezu rückstandsfrei global entgrenzt, 
docken hiermit - vermittels gewisser optischer und akustischer Ähnlichkeit zu den huzulischen Naturtrompeten - an einer wiederum lokal definierten, nun allerdings ukrainischen Schnittstelle an. Zugleich substituieren sie diese einerseits durch den Rest an Südafrikanischem, der ihnen nach wie vor innewohnt, andererseits durch ihre radikal globalisierte und globalisierende Eigenschaft.

Viele der beschriebenen Wagen und Menschen reisten am 15.1.2014 von Vaškivci in die Provinzhauptstadt Černivci, um dort mit den Feiernden aus anderen bukowinischen Dörfern zusammenzutreffen. Das Czernowitzer Malanka-Festival wurde 2014 u.a. von der internationalen Stiftung des ukrainischen Oligarchen Dmitro Firtaš finanziert. In den zurückliegenden Jahren hatte es sich in logistischer Hinsicht zusehends professionalisiert. FolkloreKollektive und -Theatergruppen wurden von den Festivalbühnen über große Bildschirme Tausenden Zuschauern auf den Plätzen der Stadt übertragen.

Auffällig ist, dass diese traditionellen Ensembles in den Tagen zuvor im Dorf Vaškivci völlig gefehlt hatten. Entgegen der auch in der Ukraine verbreiteten Auffassung, die traditionelle ukrainische Kultur sei in den Dörfern zu finden, erwies sich Malanka in Vaškivci als lockere Kompilation diverser globalisierter und traditioneller Anleihen der dörflichen Akteure unter sich. Der Fokus lag dabei deutlich auf Partymusik ohne gesteigertes Lokal- oder Traditionsbewusstsein. Anders im repräsentativ organisierten und auf touristische Außenwirkung bedachten Malanka-Festival in der Hauptstadt Černivci: Neben die Korsi derselben Dorfkarnevalswagen und ihre populären Musiken setzte sich hier ein Element inszenierter lokaler und traditioneller Authentizität - das im Dorf Vaškivci zwei Tage zuvor ganz offenbar niemand für notwendig befunden hatte.

\section{d) Korrektive Beobachtungen}

Die hier rekapitulierte Feldforschung stützt sich in erster Linie auf die Dokumentation von Feiertagen. Das macht sie potenziell anfällig für Wahrnehmungsfehler und Verzerrungen. Ihr Befund der stilistischen und die Verortung betreffenden hohen Diversität und Flexibilität von Programmdispositionen, Musizierpraktiken und Hörgewohnheiten stimmt indes mit Beobachtungen außerhalb von Feiertags-Arrangements überein: etwa der Musikauswahl in Karaoke-Bars (dokumentiert insbesondere in der KaraokeBar Pub34 im westukranischen Černivici über einen Zeitraum zwischen Sommer 2013 und Winter 2015) und den Aussagen befragter Ukrainer über ihre Musikpräferenzen. 
So wurde zwar in der Kararoke-Bar zu keinem Zeitpunkt genuin traditionelle ukrainische Musik gewünscht. Wohl aber wechselten russische (quantitativ dominierend) und ukrainische Popsongs, von denen nicht wenige folkloristische Anleihen aufgreifen. Von Seiten der Bar gab es AnimationsSänger, die dann zum Einsatz kamen, wenn an den Tischen keine Musikwünsche gemeldet wurden. Sie sangen überwiegend angloamerikanische Klassiker, etwa von den Beatles oder Elvis Presley. Wenn weder KaraokeTapes angefordert wurden noch Animations-Sänger im Einsatz waren, liefen per default Elektro Swing-Tapes des Berliner Künstlers Parov Stelar in verminderter Lautstärke.

In qualitativen Interviews mit Ukrainern wurde traditioneller ukrainischer Musik eine herausragende Rolle bei der Selbstdefinition und -identifikation als Ukrainer auch dann zugesprochen, wenn diese selbst nicht Teil der aktiven Hörgewohnheiten der Befragten war. Sehr oft wurden ukrainische Rockbands wie Okean Elzy oder der (im Februar 2015 bei einem Autounfall verstorbene) Rocksänger Kuz'ma Skrjabin genannt - neben klassischen angloamerikanischen Bands wie Nickleback, Metallica, Red Hot Chili Peppers etc. Auch die im Ausland bekannteste deutsche Band Rammstein erfreut sich einiger Beliebtheit.

Nicht selten wurde auf ukrainische Crossover-Bands wie Dachabracha oder den Musiker Oleh Skrypka verwiesen, die traditionelle Elemente in ein zeitgenössischeres, mithin anschlussfähigeres Setting transferieren. Dies wird oft sehr positiv gesehen und teils mit großem Interesse verfolgt. Auch diejenigen, die befürchten, globalisierte Hörgewohnheiten könnten der traditionellen ukrainischen Musik den Rang ablaufen, stimmten darin überein, dass bei Anlässen, in denen eine lokale Selbstidentifikation oder ein traditioneller Bezug anstehe, die Relevanz dieser Musik generationsübergreifend gesichert sei.

Mit diesen Beobachtungen kann ein potenziell verzerrender Effekt der spezifischen Feiertags-Situation zumindest relativiert werden. 


\section{Forschungszyklus $2014 / 15$ (nach Ausbruch des bewaffneten Konflikts)}

Der Forschungszyklus 2014/15, nach Ausbruch des bewaffneten Konflikts, zeigte teilweise weitreichende Verschiebungen in der Programmierung und Realisierung der identischen Festtage.

\section{a) L'viv, Nationalfeiertag $(24.8 .2014)$}

L'viv war am 24.8.2014 wie nahezu das gesamte Land in ein Meer ukrainischer Flaggen verwandelt. Mit Rücksicht auf den Krieg wurde indes auf ein offizielles musikalisches Programm vollständig verzichtet. Eine Bühne gab es nicht. Die einzige Ausnahme war ein Flashmob auf allen Plätzen der Stadt gleichzeitig, zum gemeinsamen Singen der Nationalhymne. Auf dem Marktplatz wurde die Nationalhymne gleich zweimal hintereinander gesungen, unterbrochen von emphatischen Wechselrufen »slava Ukraïni - »herojam slava (»Hoch lebe die Ukraine / Ehre der Ukraine« — »Ehre den Helden«). War dieses Antiphon in patriotischen Kreisen insbesondere in L'viv schon seit jeher ein Ausweis besonderen Nationalbewusstseins gewesen, so ging es im Zuge der Proteste auf dem Kiewer Majdan Nezaležnosti ab Ende 2013 in die kollektiven Rituale nationaler Selbstidentifikation über. Aus diesen ist es seither nicht mehr entschwunden, es brandete auch im weiteren Verlauf des Feiertags immer wieder auf (vgl. auch einschlägige YouTube-Kommentare unter beinahe beliebigen ukrainischen Musikvideos). ${ }^{7}$

An den Lemberger Nationalhymnen-Flashmob schlossen sich lückenlos weitere, nicht offiziell vorgesehene Gesänge an. Zunächst erklang beinahe obligatorisch das extrem populäre »Červona Ruta« (»Rote Raute« [=Strauch]). Der Song wurde 1968 von dem Czernowitzer Medizinstudenten Volodymyr Ivasjuk (1949-1979) komponiert und fand dann u.a. durch die Coverversion Sofija Rotarus in der gesamten Sowjetunion Verbreitung. Nicht zuletzt die Tatsache, dass der Originalsong nie urheberrechtlich geschützt

7 Beispielhaft Kommentare unter einer Okean Elzy-Kompilation (Slušaenko et al. 2014f.): [...] Petr Joha: »GLORY TO UKRAINE! « - Ivan Prostoy: »Das ist das Gewissen und die Seele der Ukraine. In unseren Seelen gibt es keine Aggression und keinen Krieg! Friede Eurem Hause !!!« - Aleksandr Golofaev: »Es kann der lobenden Worte nicht genug geben. Slava und noch einmal Slava Ukraine « [...] Vladimir Ageyev: "Super Gruppe und großartige Lieder. Slava Ukraine!!! weicht nicht zurück und gebt nicht auf!!« - [...] Elena Slušaenko: »Danke für das großartige Album! Slava Ukraine! « - Antwort Dr. Arcady Kotler: »Gerojam Slava!« - [...] Serg Palladin: "Slava Ukraïni!« - [...] Roman Šmigel's'kij: »VAKARČUK [Frontmann der Band] - UKRAïNA!!!!« (Übersetzung des Autors). 
wurde, hat seine Genese als erdachter und komponierter Popsong nahezu vergessen gemacht und begünstigt, dass er in der Ukraine den Status eines volksliedhaften Allgemeinguts und sogar einer zweiten, inoffiziellen Nationalhymne erlangen konnte. Die Koppelung des Lieds an die offizielle Nationalhymne lässt sich auch in anderen Zusammenhängen belegen (etwa bei Aufmärschen der rechten Svoboda-Partei in Černivci, dokumentiert im September 2014).

An »Červona Ruta« schlossen sich weiterhin nunmehr genuin traditionelle ukrainische Lieder an wie z.B. das beliebte »Pidmanula Pidvela« (wörtlich »Du hast mich getäuscht und verlassen«), das in so gut wie allen Landesteilen immer wieder auf gut gelaunten Feiern zu hören ist. Obwohl einige jüngere Leute mit Textbüchern für den weniger bekannten Wortlaut späterer Strophen vorbereitet waren und insofern auch eine gewisse Gestaltungshoheit über die Auswahl und Abfolge der zu singenden Lieder für sich in Anspruch nahmen, ist diese Darbietung inmitten des versammelten Menschenauflaufs als spontan und kollektiv anzusehen, eine Dissoziation in Darbietende und Rezipierende fand keineswegs statt. Die Darbietung brach auf ihrem Höhepunkt zugunsten einer spontanen Schweigeminute für die im Osten gefallenen Landsleute ab.

Interessant ist, dass inmitten der traditionellen Lieder von einigen aus der Menschenmenge angeregt wurde, auch Songs von Okean Elzy zu singen, einer der populärsten Rockgruppen des Landes. Auch wenn dies vom Kollektiv nicht in die Tat umgesetzt wurde, legt es den Schluss nahe, dass nicht nur einige traditionelle ukrainische Lieder die Nationalhymne gleichsam ideell fortzuschreiben in der Lage sind. Vielmehr kann offenbar auch bestimmte Rockmusik einen vergleichbaren Grad nationaler Identifikation kolportieren wie Nationalhymne und traditionelle Lieder. ${ }^{8}$

8 Es ist in diesem Zusammenhang daran zu erinnern, dass so gut wie alles, was in der ukrainischen Pop- und Rockszene Rang und Namen hat, irgendwann während der lang anhaltenden Proteste auf dem Kiewer Majdan konzertiert hat z. B. Okean Elzy, Nina Matvienko, Marija Burmaka, Oleksandr Ponomarëv, TNMK, Hajdamaky, Kozak System, Tartak, Plač Eremiï, Mandry, Dakh Daughters, Dachabracha; die bekannteste ukrainische Popsängerin Ruslana Lyžyčko verbrachte 100 Tage bei täglichem Gesang auf dem Majdan. Weitere Musiker waren anwesend, traten aber bewusst nicht musikalisch in Erscheinung $-z$. B. Oleh Skrypka, Ilja Jeresko, Andrij Hlyvnjuk. Wieder andere verfassten Songs zur Unterstützung der Majdan-Proteste - z. B. Ljapis Trubeckoj, der Rapper Vožyk. Mehrere namhafte Popmusiker haben einen Abgeordnetensitz in der nachrevolutionären rada (Parlament) - z.B. die Eurovisions-Teilnehmerin des Jahres 2013 Zlata Ognevič (Radikale Partei), der Sänger und Universitätsrektor Mychajlo Poplavs'kyj (parteilos). Die Popsängerin und ehemalige ukrainische Kulturministerin Oksana Bilozir verfehlte ihren Wiedereinzug für den Blok Petro Porošenko. Deputierte früherer Parlamente waren u.a. Ruslana (2006-2007), der 


\section{b) Sachnovščyna und Lozova, Stadtfeste}

(17. bzw. 16.9.2014)

Auch in Sachnovščyna fand das Stadtfest 2014 nur in radikal verkürzter Form statt. Nicht nur auf die Stände der Dörfer zwischen Kulturpalast und Leninplatz verzichtete man ersatzlos. Auch die Militärkapelle gab es nicht, selbst nicht zur Parade und Blumenniederlegung vor dem Denkmal der gefallenen Soldaten. Statt der sowjetischen Märsche des Vorjahres tönte während der gesamten ca. halbstündigen Gedenkzeremonie Robert Schumanns »Träumerei« in einer sphärischen synthetischen Chorversion in einem Endlos-Loop.

Das Konzert im Kulturpalast ähnelte in der Mischung folkloristischer Kindertanzdarbietungen, russischer und ukrainischer Popcovers, dem Auftritt eines professionellen ukrainischen Folklore-Ensembles sowie einer ukrainischen Metal-Formation der Programmierung des Lemberger Nationalfeiertags 2013. Offenbar hatte man sich in Sachnovščyna bewusst entschieden, trotz der schmalen Programmgestaltung auf Vielfalt erst recht nicht zu verzichten. Ebenso bewusst schien indes die Entscheidung, militärische bzw. sowjetische Musik wegzulassen, ebenso die im letzten Jahr ausgerechnet durch den militärisch-sowjetischen Klangkörper vertretene angloamerikanische Musik.

Auch im nur fünfzig Kilometer entfernten Lozova wurde 2014 eine Rumpf-Feier durchgeführt. Allerdings sind ihre gegenüber Sachnovščyna völlig anderen Gewichtungen bezeichnend. Nicht nur wurden sowjetische Märsche exzessiv sowohl von der Militärkapelle als auch von Tonband gespielt u.a. "Gimn Voenno Vozdušnih Sil SSSR « (die Hymne der sowjetischen Luftwaffe), »Tri Tankista« (»Drei Panzersoldaten«). Auch schritt die Prozession prominent unter SSSR-, Rote-Armee- und Lenin-Flaggen. Das ansonsten gebräuchliche gelb-schwarze Georgsband der Roten Armee hingegen fand sich 2014 erstmals durch grün-violette Bändchen in den Stadtfarben ersetzt; diese wurden angesichts der russischen Aggression - so die einhellige Sichtweise der interviewten Lozovaer - in nur 90 Kilometern Entfernung offenbar als opportuner angesehen.

Den Veteranen und ihren Angehörigen an einer großen, hufeisenförmigen Tafel reichte der Bürgermeister je eine Portion kaša (Buchweizen) und $100 \mathrm{~g}$ vodka. In einem von zwei jungen Männern in Anzug moderierten Karaoke-Wettbewerb traten anschließend in erster Linie Veteranen und reifere Frauen gegeneinander an. Die sowjetischen Soldatenschlager, die sie, nicht

Frontmann der Band Okean El'zy, Svjatoslav Vakarčuk (2007-2008) und die Popsängerin Taïsia Povalij (2012-2014). 
selten von Alter und Invalidität gezeichnet, schmetterten - u.a. »Pesenka frontova šofëra« (»Liedchen des Frontfahrers«; Vlad Nežnyj 1934), »Katjuša (Matvej Blanter / Mihajl Issakovskij 1938), "Soldaty v put'« (»Soldaten auf dem Weg«; Vasilij Solov'ëv-Sedoj / Mihajl Dudin 1954), »Idët soldat po gorodu« (»Geht ein Soldat durch die Stadt«; Vladimir Šainskij / Mihajl Tanič 1976), »Potomu, što my piloty« (»Weil wir Piloten sind«; Solov'ëev-Sedoj / Aleksej Fat'janov 1946) -, standen in augenfälligem Missverhältnis zu dem showartigen, sichtbar an einschlägigen Fernsehformaten orientierten Moderationsformat.

Zwischen allen Programmpunkten konzertierten auch in Lozova mehrere ukrainische Folklore-Kollektive, zu deren Gunsten von Band abgespielte sowjetische Märsche brutal mitten im laufenden Stück abgeschaltet wurden.

Die befragten Einheimischen empfanden auf Nachfrage das direkte $\mathrm{Ne}$ beneinander ukrainischer und sowjetischer Elemente nicht als Widerspruch. ${ }^{9}$ Auch wurde ausdrücklich keine Verbindung zwischen sowjetischen Markern und dem heutigen Russland hergestellt. Bei der Evokation der sowjetischen Insignien, so wurde klargestellt, handele es sich um eine rein retrospektive Angelegenheit im Sinne der noch lebenden Veteranen, deren Verdienste man traditionsgemäß und ohne Unterschied zu den Vorjahren gedenke. ${ }^{10}$

9 Hierbei ist zu bedenken, dass ukrainische Folklore unter sowjetischer Kulturpolitik durchaus gefördert wurde - vgl. u.a. Borys 1980.

10 Bezeichnenderweise erwies sich das in Lozova erklingende "gerojam slava a als fundamental anders kodiert als etwa in L'viv oder Korosten'. Weder ist es zu verstehen als Antiphon des im Zusammenhang mit den Majdan-Protesten etablierten, patriotischen Schlachtrufs »slava Ukraïni «, noch steht es im Bezug zu deren »Helden « (oder, historisch weiter ausgreifend, den »Helden« anti-sowjetischen Widerstands um Stepan Bandera). Dasselbe Mem, das in L'viv in ritueller Selbstvergewisserung ein ums andere Mal die Unabhängigkeit von der Sowjetunion und dem russischen Aggressor bekräftigen soll, artikuliert in Lozova die Verbundenheit mit den Veteranen der Roten Armee.

Auch im fünfzig Kilometer von Lozova entfernten Sachnovščyna war nur das allein stehende »gerojam slava«, nicht das Lemberger Wechselwort, in der Bevölkerung habitualisiert. Dem Bürgermeister selbst blieb es überlassen, zum Abschluss seiner Rede das vorgesehene »slava gerojam « mit »slava Ukraïni $z u$ komplementieren, es somit zugleich der sowjetischen, retrospektiven Kontextualisierung zu entreißen und als Ausdruck patriotischen Ukrainertums neu zu kodieren. Dazu passt, dass der parteilose Bürgermeister von Sachnovščyna die Zeremonie überwiegend auf Ukrainisch stattfinden ließ. In der Nachbarstadt Lozova, das von einem Bürgermeister der 2014 aufgelösten Partija Regioniv (Partei der Regionen) Vyktor Janukovyčs regiert wird, wurde der größere Teil der Zeremonie in russischer Sprache gehalten. - Auch auf der Ebene der Sprache ist von einem binären Ost/West-Schisma nicht viel Leistungsfähigkeit zu erwarten. Vielmehr gilt, ähnlich wie bezüglich der musikalischen Präferenzen und Praktiken, gerade in Regionen des Ostens eine weitreichende Toleranz und Kompatibilität russischer und ukrainischer Sprache. Deren Prävalenz werden ansonsten auch von kleinmaschigen Faktoren wie Urbanität (russischsprachig) 


\section{c) Korosten', Kartoffelpufferfest (13.9.2014)}

Der Kartoffelpuffer-Feiertag in Korosten' wurde aufgrund des Kriegs von der Stadtverwaltung ganz abgesagt. Er fand lediglich in einer privaten Feier außerhalb der Stadt einen geringfügigen Ersatz. Während im Vorjahr Stände und Folklore-Ensembles im Zentrum Korosten's den Feiertag bestimmt hatten, war 2014 einzig für den Abend eine Rockband aus der Provinzhauptstadt Žytomyr eingeladen. Notabene konzertierte diese nicht in der (nach dem Atomunglück in Čornobyl' und dem Zusammenbruch der Sowjetunion von Strukturproblemen und Leerstand der überdimensionalen industriellen Infrastruktur betroffenen) Stadt Korosten', sondern quasi in freier Natur: Die Siedlung Vil'ne ist nicht mehr als ein paradiesisch anmutender Landstrich am Fluss mit einigen in traditionellem ukrainischen Baustil errichteten Häuschen, halb Freilichtmuseum, halb Hippie-Kommune. Etwa hundert teils traditionell angezogene Menschen hatten sich im Laufe des Tages dort zum Feiern eingefunden, überwiegend Bekannte aus dem Umfeld des Erbauers und Gurus des Museumsdorfs, Hryhorij Hryhorevyč Pašinskij.

Die geladene Rockband spielte zumeist eigene Kompositionen. Ein Cover der inoffiziellen ukrainischen Nationalhymne bzw. des 'falschen Volkslieds „Červona Ruta« fehlte genauso wenig wie ein Rock-Cover des sechten< traditionellen Liedes »Pidmanula Pidvela« - wie bei den spontanen Gesängen der Menschenmenge in L'viv. Ganz offenbar durch diesen sich zuspitzenden Rekurs auf traditionelles oder als solches empfundenes Liedgut geriet das mitsingende und -tanzende Publikum in einen Zustand patriotischer Mobilisierung. In der an das »Červona Ruta «-Cover anschließenden Pause vor dem nächsten Song entluden sich frenetische »Putin chujlo«-Sprechchöre, die der Schlagzeuger der Band mit unverkennbaren Trommelwirbeln würdigte (während die restlichen Bandmitglieder sich unbeteiligt gaben).

»Putin chujlo« ist im Zuge des bewaffneten Konflikts in der Ostukraine, der von der ukrainischen Bevölkerung großenteils als Aggression Russlands verstanden wird, zu einem Mem der nationalen Selbstidentifikation in der Ukraine geworden. Eine wörtliche Übersetzung ist schwierig, wobei chuj ein der ukrainischen und russischen Vulgärsprache mat' entstammender, sehr unflätiger Ausdruck für Penis ist. Meist wird chujlo als »Schwanzgesicht « (engl. »dickface«) übertragen. Hinter dem Sprechchor steht eine simple musikalische Mikroform, auf die der Schlagzeuger mit den einschlägigen Wirbelrhythmen verweisen konnte. Es handelt sich ursprünglich um einen

und Ruralität (teilweise ukrainischsprachig) sowie politischer Zugehörigkeit beeinflusst. 
Hooligan-Song aus dem Umfeld des ostukrainischen Fußballvereins Metalist Charkiv, der sich in der originären Version gegen den Präsidenten des ukrainischen Fußballbundes (2000-2012), Hrihorij Michajlovyč Surkis, richtete (Surkis chujlo). Der Chorus wird durch stampfende Trommelwirbel eröffnet und begleitet und umfasst außer dem skandierten Surkis bzw. Putin chujlo eine dreifach absteigende Sequenz auf die Silben »la la la la la la la laaa«.

Am 30.3.2014, einen guten Monat nach Krym-Annektion und Beginn prorussischer Proteste ${ }^{11}$ in verschiedenen Gegenden der Ostukraine, wurde die Neutextierung zugunsten von »Putin chujlo « erstmals belegt, auf einem gemeinsamen Aufmarsch von Fans der Vereine Metalist Charkiv und Šachtar Doneck. Inzwischen ist der Chorus völlig vom ostukrainischen HooliganMilieu entkoppelt und auf dem gesamten ukrainischen Territorium kurrent.

11 Größere pro-russische Proteste gab es außer im späteren Kriegsgebiet Doneck und Luhans'k u.a. auch in der zweitgrößten ukrainischen Stadt Charkiv, in Dnipropetrovs'k und in Odesa. Die Bewertung dieser Proteste ist innerhalb der Ukraine sowie international umstritten. Russland und Majdan-kritische Stimmen in der Ukraine sehen die Ursache der Proteste in der Bevölkerung und in deren Ablehnung der postrevolutionären Regierung. Sie verweisen u.a. auf einen unmittelbar nach dem Umsturz am 23.2.2014 von der verchovna rada (Parlament) beschlossenen, allerdings niemals in Kraft getretenen Gesetzesentwurf, der den Status von Sprachen ethnischer Minderheiten als zweite regionale Amtssprache (in Oblasten mit mehr als 10\% Bevölkerungszugehörigkeit zu dieser Sprachgruppe) hätte abschaffen sollen, ferner auf die Rolle des pravij sektor (Rechter Sektor) und anderer nationalistischer Kräfte während des Umsturzprozesses sowie die fragwürdige demokratische und völkerrechtliche Legitimierung der postrevolutionären Übergangsregierung unter Ministerpräsident Arsenij Jacenjuk. Unbestritten ist der starke Einfluss russischsprachiger Medien aus Russland in der Ostukraine. Diese befeuerten Ängste der regionalen Bevölkerung vor der angeblich »faschistischen Kiewer Junta « - stellvertretend Pravda vom 5. März (Anon. 2014a) -, bis zu einem vermeintlich bevorstehenden antirussischen »Genozid « - stellvertretend Jurij Kotenok (2014) vom 24. April. Ebenso unbestritten ist, dass sich eigens eingereiste Menschen aus Russland - in Charkiv bspw. überwiegend aus dem jenseits der nahen Grenze gelegenen Belgorod, aber auch aus Moskau - bereits im März 2014 an den Protesten beteiligten.

Die Ukraine, die EU, die USA und die NATO gehen davon aus, dass Russland nicht nur den Zustrom russischer Hooligans und Krawallmacher nicht unterbindet, sondern darüber hinaus Spezialeinheiten und spätestens seit August 2014 Waffensysteme und reguläre Soldaten auf ukrainisches Territorium einschleust. Russland dementiert dies und behauptet, die gefangengenommenen oder gefallenen russischen Soldaten seien in ihrem Urlaub und auf eigenen Antrieb oder versehentlich in die Ukraine eingereist. Angehörige (Žilin 2014) und die Vereinigung der Soldatenmütter in Russland (Sojuz Komitetov Soldatskih Materej Rossii), die diese Theorien bestreiten und u.a. eine finanzielle Entschädigung von der Armee fordern, sehen sich laut eigenen Aussagen Repressalien ausgesetzt (Anon 2014b; Tumanov 2015). Die Vereinigung der Soldatenmütter Sankt-Petersburg beispielsweise wehrt sich erfolglos gegen den ihr am 28.8. 2014 zugewiesenen Status eines »ausländischen Agenten« gemäß des 2012 von Vladimir Putin erlassenen Gesetzes »Ob NKO (»Über NGO«). 
Sogar ein von ukrainischen Astronomen entdeckter und benannter Asteroid hört inzwischen auf den Namen Putin chujlo. Auf YouTube oder in der ukrainischen Wikipedia findet man zahllose Aufnahmen und Cover-Versionen von Bands, die sich - anders als die in Korosten' auftretende Gruppe - bei ihren Konzerten einer vollständigen Darbietung des Songs nicht enthielten. Dabei wird dem Song von einem Uploader das Label einer »ukraïns'ka narodna pisna«, also eines ukrainischen Volkslieds, zugeordnet (hrendyabliki 2015).

Dies ist umso pikanter, wenn man der nicht unplausiblen Annahme folgt, dass die Hooligan-Melodie ihrerseits ein Remake der »Speedy Gonzales «Musik von David Hess (David Dante 1961) sei (etwa Anon. 2014c). Mit deren nicht-textierten Teil stimmt sie in der Tat vollständig überein. Mithin erweist sich der in Korosten' von den Zuhörenden intonierte Chorus »Putin chujlo « als Folgendes: ein ursprünglich je nach Perspektive völlig anders (nämlich angloamerikanisch) oder schlicht nicht kodiertes (globalisiert kurrentes) Element, das über mehrere Stufen der lokalen Neukodierung am Ende auf dem diffusen Status eines ukrainischen »Volkslieds« mit starkem lokalem Identifikationspotenzial angelangt ist.

\section{d) Vaškivci, Malanka-Fest (13./14.1.2015)}

Wie das Kartoffelpufferfest 2014 in Korosten', so wurde auch in Černivci das offizielle Malanka-Festival 2015 kurzfristig abgesagt, wegen einer Bombendrohung. Im Vorjahr waren am 15.1.2014, nach der eigentlichen MalankaNacht am 13./14.1., Karnevalswagen aus zahllosen umliegenden Dörfern nach Černivci geströmt, um in langen, durch Absperrungen vom Publikum freigehaltenen Korsos durch die Stadt zu fahren, während auf einer großen Bühne auf dem Czernowitzer Philharmonie-Platz Folklore-Ensembles auftraten. 2015 trieben sich nur vereinzelte vermummte Gestalten in der Stadt herum und versuchten mit Waffenattrappen, Autos anzuhalten und Geld einzufordern (Musik machten sie keine).

Das Dorf Vaškivci dagegen feierte Malanka auch am 13./14.1.2015 völlig unbehelligt. Unter den Karnevalswagen befanden sich 2015 einer unter arabischem und einer unter US-amerikanischem Motto. Von letzterem, Moulin Rouge genannt, wurden Marilyn Monroe, »Sex Bomb « usw. abgespielt. Russische und ukrainische Pop- und Folkpopmusik erklangen zum Teil aus denselben Boxen und - wie in Lozova - wechselte man mitunter zum nächsten Titel, ohne das Ende des vorigen Songs abzuwarten.

Erstaunlicherweise verzichteten die Karnevalswagen im Gegensatz zu 2014 nahezu völlig auf explizite politische Botschaften (politische State- 
ments wären im Rahmen von Malanka ganz offenkundig möglich und opportun und wurden im Vorjahr 2014 auch getätigt). Allerdings erklangen 2015 zwischen unterschiedlichster Popmusik deutlich mehr traditionelle Lieder und Popcovers traditioneller Lieder als im Vorjahr. So kehrte die Koljade (Weihnachtslied) »Dobrij vičer« (»Guten Abend«) über die gesamten Feierlichkeiten hinweg immer wieder, in immer anderen stilistischen Realisierungen: im Sinne des Dreikönigssingens unbegleitet von Kleingruppen von Kindern vor Süßigkeiten verheißenden Hauseingängen; in der wohl traditionell authentischsten Form von Frauengruppen mittleren und höheren Alters und mit charakteristischem scharfen Stimmklang; in mehreren Pop-Coverversionen aus den Boxen, dann meist mit durchgehendem Beat etwa auf das anderthalbfache Tempo hochgepeitscht. Manche der Popversionen, typischerweise mit Sologesang, öffneten sich in rückblendeartigen, beatfreien formalen Fenstern zu Einspielungen der authentischen traditionellen Frauenchöre, leisteten mithin innerhalb der entgrenzenden Popbearbeitung eine reflexive Rückbindung an die ihnen zugrundeliegende traditionelle Verortung.

\section{Thesen}

Folgende Thesen lassen sich aus den dokumentierten und beschriebenen Befunden der Feiertage in L'viv, Sachnovščyna, Lozova, Korosten', Vaškivci und Černivci ableiten:

1) Traditionelle Musik ist ein zentrales Medium lokaler und nationaler Selbst-Identifikation als Ukrainer. Neben weiteren Codes wie Sprache, Flaggen, (traditioneller) Kleidung etc. ist traditionelle Musik direkt mit der lokalen Verortung verknüpft.

2) Trotz anderslautender Befürchtungen ${ }^{12}$ selbst seitens Einheimischer scheint traditionelle Musik in verblüffender Weise kompatibel mit sonstigen, anders oder nicht spezifisch lokal markierten, sondern globalisierten musikalischen Elementen und Praktiken. Dies entspricht differenzierenden Befunden in anderen Kontexten von Globalisierungs- und Glokalisierungsprozessen (vgl. u.a. Robertson-von Trotha 2009).

12 »Globalisierung, Nivellierung, Vergessen und Verluste « konstatiert die Alfred Töpfer Stiftung F.S.V. (2000: 23) in der Besprechung des von ihr 2000 für den Europäischen Preis für Volkskunst nominierten Folkloreensembles in Petrovo / Pyjterfolvo / Tiszapéterfalva (Zakarpats'ka Oblast', Südwestukraine). - Vgl. außerdem UNESCO 2003. 
3) Dabei beschränkt sich die Kompatibilität nicht auf das bloße Nebeneinander in repräsentativen Programmen sowie privaten Hörgewohnheiten. Vielmehr ergeben sich spannungsvolle und selektive Verschränkungen von unterschiedlich lokal kodierten und entgrenzten Musikelementen.

4) Die Zuordnungen und Provenienzen können dabei ganz erheblich verschwimmen und mehrfach überschrieben werden: So können komponierte Popsongs - unter weitreichendem Verlust des Bewusstseins ihres auktorialen Gemachtseins - den analogen Stellenwert althergebrachter Volkslieder erreichen (am deutlichsten »Červona Ruta«, ansatzweise Okean Elzy). Umgekehrt können Popadaptionen an die Stelle traditioneller Vorlagen treten und diese gleichsam idiomatisch ersetzen. Bestehende lokale Markierungen können dekonstruiert, nivelliert und neu kodiert werden (etwa Trembiten / Vuvuzelas, »Speedy Gonzales« / »Putin chujlo«).

5) Die Markierungen und Zuordnungen erfolgen nicht selten unscharf und fehlerhaft. Dies gilt nicht nur für nicht-ukrainische oder globalisierte Elemente, sondern auch für ukrainische. So ergibt es sich einerseits, dass Elemente unterschiedlicher lokaler Signifikanz zu diffusen Kategorien (»westlich«, »world«, »exotisch«, »traditionell«) verschwimmen. Andererseits gründen vermeintlich distinkte lokale Markierungen auf faktisch unzureichenden, fehlerhaften oder hybriden Kodierungen (z. B. Zymbal als scheinbar begleitendes Element traditionellen ukrainischen Gesangs). Dazu gehört auch, dass die Kategorie der »Folklore recht unspezifische Verwendung findet für unterschiedlichste Ausprägungen traditioneller und regionaler Referenzen (z.B. Folklore-Tanzensembles in L'viv).

6) Lokale Markierungen können selektiv an bestimmten Parametern der musikalischen Darbietung ansetzen. So kann etwa ein Popsong ohne sonstige Transformation in traditionellen Kostümen dargeboten werden. Umgekehrt kann eine traditionelle Melodie in ein lokal unspezifischeres populäres Setting (Pop, Rap) emigrieren. Unterschiedliche Genres sind unterschiedlich explizit lokal markiert oder indifferent (vgl. H. Huber 2011: 10; M. Huber 2011: 50f.). Der Meta-Kategorie des Genres kommt über diese Verknüpfung direkt eine lokalisierende bzw. entgrenzende Eigenschaft zu.

7) Als Ergebnis des vielfältigen interaktiven Geflechts lokaler Markierungsund Entgrenzungsoptionen spielt Musik des Crossovers und weitere Hybridbildungen in der Ukraine eine zentrale Rolle (vgl. hierzu u.a. Hall 1999). 
8) Zu den miteinander kompatiblen musikalischen Elementen zählen neben angloamerikanischem Pop nicht nur der quantitativ weitaus relevantere russische Pop, sondern in bestimmten Landesteilen und Kontexten auch sowjetische Militärmusik und Kriegslieder. Darüber hinaus verdanken einige der nach wie vor populärsten ukrainischen Popsongs ihre Beliebtheit nicht zuletzt ihrer weiten Verbreitung innerhalb der Sowjetunion (z. B. Sofija Rotaru).

9) Das Paradigma eines kulturellen und die Identität betreffenden Ost/ West-Schismas lässt sich anhand der Befunde und insbesondere hinsichtlich der Teilhabe an ausdrücklich ukrainischer traditioneller Musik nicht hinreichend belegen. Selbst in Gegenden bzw. Kontexten, in denen sowjetische Musik (s. 8.) und weitere sowjetische Insignien starke Relevanz besitzen, fungieren mitunter dieselben traditionellen ukrainischen Lieder als lokale Marker wie in westlichen Landesgegenden.

10) Eine Korrelation von angloamerikanischer Musik mit einer politisch-kulturellen Ausrichtung am >Westen< und russischer oder gar sowjetischer Musik am >Osten< scheint unterkomplex, da nicht zuletzt gerade im Osten beides direkt koexistiert. Eher noch scheint sowjetische Musik als lokal nicht markiert wahrgenommen zu werden, ähnlich wie globalisierte Musik westlicher Prägung. Nicht zuletzt vor dem Hintergrund weitreichender historischer, wirtschaftlicher und persönlichen Wechselbeziehungen mit Russland ist ohnedies von einem transnationalen und transkulturellen (vgl. Hühn et al. 2010) Raum speziell in den östlichen Gebieten der Ukraine auszugehen, dessen Praxis allerdings durch die jüngsten politischen Entwicklungen auf einigen Ebenen (v.a. der transstaatlichen) dramatischen Veränderungen unterworfen ist.

11) Inwieweit entweder eine lokale (Anders-)Markierung oder aber eine globalisierende Entgrenzung eingelöst wird, entscheidet sich in vielen Fällen am Einzelfall und in Abhängigkeit der Rezeptionsrichtung. So werden z.B. sowjetische Musik oder Rap je nach Zugehörigkeit zum jeweiligen Kulturkreis als vermeintlich universelle, ortlose Kategorien angesehen, oder aber als distinktes Merkmal der jeweils fremden Kultur. ${ }^{13}$

Vorsichtige Rückschlüsse aus der komparativen Betrachtung vor und nach Ausbruch des bewaffneten Konflikts ergeben:

1) In manchen Orten (Nationalfeiertag L'viv 2014, Stadtfest Sachnovščyna 2014, evtl. Malanka Vaškivci 2015) lässt sich infolge des Konflikts ein gesteigertes Festhalten an traditionellen Musikdarbietungen ausmachen.

13 Zur Mehrgleisigkeit und Wechselseitigkeit von Globalisierungsprozessen vgl. u.a. Burkhalter 2011. 
Dies ergibt sich oft (L'viv, Sachnovščyna) aus dem Weglassen anderer, im Vorjahr noch koexistenter Elemente (angloamerikanischer Pop und ukrainischer Pop in L'viv, sowjetische Musik und militärische Bigbandmusik in Sachnovščyna).

2) Es bleiben dessen ungeachtet weiterhin Musiken derjenigen lokalen Markierungen zueinander völlig kompatibel, welche nun kriegerischen Konfliktparteien zugeordnet werden könnten: etwa ukrainische Folklore und sowjetische Märsche bzw. Kriegschansons (in Lozova), oder ukrainischer und russischer Pop (in Vaškivci). Dies stimmt mit der Beobachtung überein, dass trotz der in Interviews zum Ausdruck gebrachten starken Ablehnung gegenüber russischer Ukrainepolitik und ggf. auch Russlands oder der russischen Sprache auch 2015 russische Popmusik z. B. in Karaoke-Bars ohne jede Verminderung konsumiert wird.

3) Institutionalisierte Feiertage (Nationalfeiertag L'viv 2013, Stadtfeste Sachnovščyna und Lozova 2013 und 2014, Malanka Černivci 2014) scheinen zu Folklore-Darbietungen zu neigen, insbesondere unter Verwendung professioneller oder semiprofessioneller Ensembles. Stärker private (Korosten' 2014) und nicht auf Repräsentation ausgelegte Feiertage (Vaškivci 2014 und 2015, im Gegensatz dazu Černivci 2014) scheinen demgegenüber mitunter vordergründig Pop- und Rockmusik zu bevorzugen. Dessen ungeachtet, können traditionelle und lokale Elemente starke Berücksichtigung finden (Korosten' 2015, Vaškivci 2015). Durch die Kriegssituation werden institutionalisierte Feiertage durch private Ersatzveranstaltungen (Korosten' 2014) oder spontane Aktionen im Rahmen institutioneller Rumpfveranstaltungen (Nationalfeiertag L'viv 2014) ersetzt.

4) Im Falle von Malanka in Černivci vs. Vaškivci zeigt sich auch ein StadtLand-Gefälle, wobei entgegen der Intuition und verbreiteten Annahme auf dem Land der Musikgeschmack insbesondere der jungen Leute weniger auf die Pflege der traditionellen Musik bedacht ist als in der Stadt. Diese Beobachtungen müssen allerdings zusätzlich vor dem Hintergrund der Frage nach dem in Stadt und Land u. U. verschiedenen Grad der Institutionalisierung (3.) bewertet werden. ${ }^{14}$

14 Eine dialektische Struktur von Urbanität vs. Ruralität bestimmt u.a. das Musikvideo zu dem Song »Vesna« (»Frühling«) von Dachabracha: Die traditionell gekleideten Musiker wandern durch einen karpatisch anmutenden Wald, bis sie (was geographisch unmöglich ist) in Kyïv ankommen, wo sie, Fremdkörper inmitten des hektischen Verkehrs- und Berufsgeschehens der globalisierten Metropole, durch ihre Musik aus dem Wald die Menschen zum überraschten Innehalten verleiten, in welchem die Musik magischen Besitz von deren Körpern ergreift (Dachabracha 2011). 
5) Die beherrschende Veränderung von 2013/4 zu 2014/5 ist diejenige, dass Musik im Rahmen von Feiertagen ganz grundsätzlich nur unter bedeutend schmaleren Rahmenbedingungen stattfindet. Dies mindert nicht zwangsläufig die Rolle, die der Musik qualitativ nach wie vor zukommt. Gleichwohl werden Musikdarbietungen und Feierlichkeiten gleich welcher Art und Verortung im Angesicht des Krieges als problematisch empfunden.

\section{Literatur}

Alfred Töpfer Stiftung F.V.S. (2000). Verleihung des Europa-Preises für Volkskunst. Teil: 1998/2000. Hamburg: Alfred Töpfer Stiftung.

Andruchovyč, Juri (2003). »Carpathologia Cosmophilica. Versuch einer fiktiven Landeskunde.« In: Ders., Das letzte Territorium. Essays. Frankfurt/M.: Suhrkamp, S. 38-50.

Andruchovyč, Juri (2004). »Mitteleuropäisches Memento.«In: Juri Andruchovyč / Andrzej Stasiuk, Mein Europa. Zwei Essays über das sogenannte Mitteleuropa. Frankfurt/M.: Suhrkamp (Originalausgabe 2000), S. 59-74.

Anon. (2014a). »Norvežskij Ekspert potreboval razorvat' diplomatičeskie otnošenija s fašistskoj chuntoj Kieva« [»Norwegischer Experte fordert den Abbruch der diplomatischen Beziehungen mit der faschistischen Kiewer Junta«]. In: Pravda vom 5. März, http://www.pravda.ru/news/world/05-03-2014/1197311-nistad-0 (Zugriff am 22.7.2015).

Anon. (2014b). »Zakonodatel'naja vojna $s$ >vnutrennimi vragami<. Repressii protiv rossijskih NKO « [»Der mit den Mitteln der Gesetzgebung geführte Krieg gegen sinnere Feinde . Repressionen gegen russische NGO«]. In: Soldatskie Materi Sankt-Peterburga [Webseite der Soldatenmütter Sankt-Petersburg], Blogpost vom 10. Oktober, http://soldiersmothers.ru/novosti/novost/?tx_ttnews\%5Btt_ne ws\%5D=727\&cHash=4c47825322c6f3e2067664a38bf2c1c6 (Zugriff am 29.7.2015).

Anon. (2014c). »Pat Boone - Speedy Gonzales«. In: Putin hujlo blogspot, Blogpost vom 27. Juni, http://putin-khuilo.blogspot.com/2014/06/pat-boone-speedy-gon zales.html (Zugriff am 29.7.2015).

Borys, Jurij (1980). The Sovietization of Ukraine 1917-1923. The Communist Doctrine and Practice of National Self-Determination. Edmonton: The Canadian Institute of Ukrainian Studies (2. Aufl., Erstausgabe 1960).

Burkhalter, Thomas (2011). »Experimentelle Sounds aus Beirut. Die Musiker der Bürgerkriegsgeneration definieren >Lokalität< neu. «In: West Meets East. Musik im interkulturellen Dialog. Hg. v. Alenka Barber-Kersovan, Harald Huber und Alfred Smudits (= Musik und Gesellschaft 29). Frankfurt/M. u.a.: Peter Lang, S. 113-134.

Dachabracha (2015a). [Offizielle Bandwebseite], www.dakhabrakha.com.ua (Zugriff am 15.2.2015).

Von Jana Hurul'ova existiert ein Popmusikvideo, das sie in zwei Schnittebenen in einer ebenfalls karpatischen Waldlandschaft mit Pferden einerseits und inmitten des historischen Stadtzentrums von L'viv in urbanem Outfit andererseits zeigt (Hurul'ova 2013b). 
Dachabracha (2015b). [Fanpage auf Facebook], https://www.facebook.com/Dakha Brakha/info?tab=page_info (Zugriff am 15.2.2015).

Dachabracha (2015c). [Fanpage auf Vkontakte], www.vk.com/dahabraha (Zugriff am 15.2.2015).

Hall, Stuart (1999). »Kulturelle Identität und Globalisierung.« In: Widerspenstige Kulturen. Cultural Studies als Herausforderung. Hg. v. Karl H. Hörning und Rainer Winter. Frankfurt/M.: Suhrkamp, S. 393-441.

Huber, Harald (2011). »Editorial.« In: West Meets East. Musik im interkulturellen Dialog. Hg. v. Alenka Barber-Kersovan, Harald Huber und Alfred Smudits (= Musik und Gesellschaft 29). Frankfurt/M. u. a.: Peter Lang, S. 7-18.

Huber, Michael (2011). »Detroit / Wien / Tokio. Interkultureller Dialog durch elektronische Musik? « In: West Meets East. Musik im interkulturellen Dialog. Hg. v. Alenka Barber-Kersovan, Harald Huber und Alfons Smudits (= Musik und Gesellschaft 29). Frankfurt/M. u. a.: Peter Lang, S. 49-63.

Hühn, Melanie / Lerp, Dörte / Petzold, Knut / Stock, Miriam (2010). »In neuen Dimensionen denken? Einführende Überlegungen zu Transkulturalität, Transnationalität, Transstaatlichkeit und Translokalität.« In: Transkulturalität, Transnationalität, Transstaatlichkeit, Translokalität. Theoretische und empirische Begriffsbestimmungen (= Region, Nation, Europa 62). Hg. v. Melanie Hühn, Dörte Lerp, Knut Petzold und Miriam Stock. Berlin: Hopf, S. 11-46.

Jilge, Wilfried (2005). »Nachwort.«In: Mykola Rjabčuk, Die reale und die imaginierte Ukraine. Essay. Frankfurt/M.: Suhrkamp, S. 169-176.

Kotenok, Jurij (2014). »Junta načala genocid« [Die Junta hat mit dem Genozid begonnen; Interview mit Boris Džerelievskij]. In: Segodnja.ru. Informacionno-analitičeskoe setevoe izdanie, 24. April, http://www.segodnia.ru/content/138236 (Zugriff am 24.7.2015).

Mezger, Werner (1984). Narretei und Tradition. Die Rottweiler Fasnet. Stuttgart: Theiss.

Rjabčuk, Mykola (2005). Die reale und die imaginierte Ukraine. Essay. Aus dem Ukrainischen von Juri Durkot und mit einem Nachwort von Wilfried Jilge. Frankfurt/M.: Suhrkamp.

Robertson-von Trotha, Caroline Y. (2009). Die Dialektik der Globalisierung. Kulturelle Nivellierung bei gleichzeitiger Verstärkung kultureller Differenz. Karlsruhe: Universitätsverlag Karlsruhe (Print on Demand).

Slušaenko, Elena et al. (YouTube-Nutzer) (2014f.). Kommentare unter Okean Elzy Izbranoe / Okean Elzy - The Best. Hochgeladen von MELOMAN, 14.8.2014, https://www.youtube.com/watch?v=Dp-Gzpcflj4 (Zugriff am 22.7.2014).

Tumanov, Grigorij (2015). »Sistema >Ni odnogo okna« [»Das System >keines einzigen Fensters «]. In: Soldatskie Materi Sankt-Peterburga [Webseite der Soldatenmütter Sankt-Petersburg]. Blogpost vom 21. Juli, http://soldiersmothers. $\mathrm{ru} /$ novosti/novost/?tx_ttnews\%5Btt_news\%5D=936\&cHash=edd55d2806a90b83ea 11aa6e7618fee0 (Zugriff am 29.7.2015).

UNESCO (2003). Convention for the Safeguarding of Intangible Cultural Heritage. Paris: 2003, http://www.unesco.org/culture/ich/index.php?lg=en\&pg=00006 (Zugriff am 15.2.2015).

Wissenschaftliche Dienste des Deutschen Bundestags (2010). Aktueller Begriff zur völkerrechtlichen Kategorisierung von Konflikten (= Wissenschaftliche Dienste des Deutschen Bundestags 46/10), https://www.bundestag.de/blob/191426/ 3c0cf9515fa4bdf8337d042ae2b9fc5c/kategorisierung_von_konflikten-data.pdf (Zugriff am 15.2.2015). 
Žilin, Ivan (2014): » On otdal svoju žizn', a ego privezli vot tak...< Rossijskij soldatkontraktnik Sergej Andrijanov pogib na vostoke Ukrainy. Ob etom ego devuška raskazala korrespondentu >Novoj « [»>Er hat sein Leben gelassen, und sie haben ihn so hergebracht... - Der russische Berufssoldat Sergej Adrijanov fiel im Osten der Ukraine. Darüber erzählt seine Freundin dem >Novaja<-Korrespondenten «]. In: Novaja Gazeta vom 24. November, http://www.novayagazeta.ru/society/ 66219.html (Zugriff am 29.7.2015).

\title{
Audio- / Videographie
}

Dachabracha (2011). DakhaBrakha - Vesna. Hochgeladen von DakhaBrakha am 9. Januar, https: //www.youtube.com/watch?v=T30JVMuHwcU (Zugriff am 15.2. 2015).

hrendyabliki (YouTube-Nutzer) (2014). Putin - chujlo (mega versija - power mix), hochgeladen am 11. April, https://www.youtube.com/watch?v=sjtTrup5qNU (Zugriff am 15.2.2015).

Hurul'ova, Jana (2013a). Jana Hurul'ova - Letila zozulja (ukraïns'ka narodna pisna), hochgeladen von Roman Pogribnyj am 13. Juli, https://www.youtube.com/ watch? $\mathrm{v}=\mathrm{nmv89Pt3b08}$ (Zugriff am 15.2.2015).

Hurul'ova, Jana (2013b). Jana Hurul'ova - Dytjače Jevrobačennja 2013, Oj, ty, vitre, hochgeladen von Jana Hurul'ova am 3. August, https://www.youtube. com/watch?v=_Ts0EIQ8gzw (Zugriff am 15.2.2015).

\begin{abstract}
The recent Euromaidan revolution and the ongoing armed conflict in East Ukraine are only the latest episodes in the country's struggle for national self-determination and identity. This paper explores how musical performances during Ukrainian holiday celebrations serve as a vital tool for policies of both regional and global referencing. Documentation of different festivities in West, East, and central regions of Ukraine is represented, as well as diverse infrastructural and societal framings.

Based on a detailed description of the ethnographical findings, the paper contends that conceptions of a purported schism between East and West Ukraine, and of the unilateral extinction of regional traditions through globalised pop music, need to be re-evaluated. Instead, a highly complex, if not seemingly contradictory interplay of multiple and hybrid references and local encodings are to be observed, among which traditional Ukrainian elements prominently interact with symbols of Western, global, Russian, and even Soviet connotation.
\end{abstract}

